\title{
Recommendations for medical care of oncological patients during the COVID-19 epidemic: experiences from China
}

\author{
Hong Zhao ${ }^{1}$. Shunda $\mathrm{Du}^{2}$. Jianqiang $\mathrm{Cai}^{1} \cdot$ Yilei $\mathrm{Mao}^{2}$
}

Received: 29 April 2020 / Accepted: 4 May 2020 / Published online: 12 May 2020

(C) Italian Society of Surgery (SIC) 2020

As of April 28, 2020, the confirmed cases of Coronavirus disease-19 (COVID-19) have reached 2,954,222 according to the World Health Organization's daily situation report [1]. As COVID-19 continues to spread globally, the medical systems in many countries have come under immense pressure, and medical resources are being monopolized by attempts to tackle the COVID-19 crisis. Cancer patients are often elderly and immunocompromised because of underlying malignancies and anticancer treatments and thus are at a high risk for contracting respiratory viral infections. Most hospitals have taken efforts to limit outpatient visits, reduce admissions, and cancel nonemergency operations to prevent cross-infection. Hospitals and clinicians are being more cautious with cancer patients and often cancel appointments or delay treatments for nonemergency patients. As Chinese oncologists, we conducted an online questionnaire to survey the experience and suggestions of all Chinese clinical experts in their clinical practice over the previous 2 months. We used hepatocellular carcinoma (HCC) as a representative cancer to understand clinicians' specific suggestions for patients at different cancer stages. Although this was a survey of a specific cancer, it revealed some common problems for managing cancer during the pandemic. From the Chinese experts' opinions, we summarized several suggestions to help provide the best available treatment for all cancer patients threatened by the pandemic worldwide.

Jianqiang Cai

caijianqiang@cicams.ac.cn

$\triangle$ Yilei Mao

pumch-liver@hotmail.com

1 Department of Hepatobiliary Surgery, National Cancer Center/National Clinical Research Center for Cancer/Cancer Hospital, Chinese Academy of Medical Sciences and Peking Union Medical College, Beijing 100021, China

2 Department of Liver Surgery, Peking Union Medical College (PUMC) Hospital, PUMC and Chinese Academy of Medical Sciences, Shuaifuyuan, Wangfujing, Dong-Cheng, Beijing 100730, China
First, online medical care should be actively advocated to minimize exposure at health care facilities. In the survey, most clinicians experienced a reduction of over $50 \%$ in their original workload. Most tertiary hospitals that we investigated have launched online medical consultation services. The National Health Commission, the governmental authority in China, has authorized online prescriptions, extended prescription expirations dates, and opened online drugstores during the pandemic [2]. Therefore, cancer patients can remain at home and minimize hospital visits as much as possible. Furthermore, for convenience, internet healthcare services should be encouraged in the future.

Second, noninvasive or minimally invasive procedures should be suggested for cancer patients in the early or intermediate stages, because radical operations will need to be postponed. For example, transcatheter arterial chemoembolization and radiofrequency ablation are preferred for earlystage HCC patients during the pandemic. Many oncologists also recommend oral targeted therapy as a bridge therapy for patients with resectable tumors during the waiting period. Evidence suggests that compared with observation alone, bridge therapy using sorafenib is cost effective and safe for early-stage HCC patients awaiting liver transplantation [3]. Therefore, under the pressure of the COVID-19 pandemic, reliable nonsurgical treatments are preferred over surgery. Neoadjuvant or bridging therapies should also be recommended to patients with other cancers.

Third, for patients receiving antitumor medications, oral drugs should be promoted if available. As with HCC, targeted therapy using sorafenib or lenvatinib is the first-line treatment for advanced patients with extrahepatic metastasis [4]. If first-line oral targeted therapies fail during this period, Chinese oncologists suggest combining oral targeted drugs with new immunotherapies. For patients being treated at home, internet guidance should be widely recommended to handle adverse reactions and follow-up visits. In addition, routine follow-up visits during treatment should be postponed. Patients should discuss appropriate follow-up visit 
intervals with their oncologist according to each patient's disease status and physical condition.

Finally, clinics or hospitals should only be visited under strict protective conditions when patients' problems cannot be resolved via internet consultation. Wang and colleagues reported some effective active measures for screening patients and preventing infections, which have been conducted at the National Cancer Center over the last 2 months, including on-site temperature testing at the entrance, recording contact information and travel history, and using an appointment scheduling system [5]. Under these measures, people who had potential influenza-related symptoms or had been to epidemic areas were forbidden to enter the hospital, and the appointment system reduced crowds. All patients were recommended to receive basic tests, including routine blood examinations and liver and renal function tests, at nearby clinics. More importantly, patients should take strict personal protection measures, including wearing masks and carrying their own disinfectant during the entire visit.

It is difficult to predict the extent of the influence that the pandemic will have worldwide, but oncologists should try to provide the best possible individualized and available management and therapy for all cancer patients rather than simply waiting. Fortunately, China has moved into the mitigation stage, and we believe that all countries will eventually conquer this crisis. These experiences and suggestions from Chinese oncologists may help provide a basis for valuable decision-making for oncologists worldwide according to their own medical systems.

Author contributions $\mathrm{HZ}$ and SD wrote the draft manuscript. JC and YM revised the manuscript. All authors read and approved the final manuscript.

Funding None.

\section{Compliance with ethical standards}

Conflict of interest The authors declare that they have no competing interests.
Research involving human participants and/or animals This article does not contain any studies with human participants or animals performed by any of the authors.

Informed consent All authors agree to submit for consideration for publication in Updates in Surgery.

\section{References}

1. World Health Organization. Coronavirus Disease 19 (COVID19) Situation Report-99. Apr 28, 2020. https://www.who.int/docs/ default-source/coronaviruse/situation-reports/20200428-sitrep-99covid-19.pdf?sfvrsn=119fc381_2. Accessed 29 Apr 2020

2. The general office of the National Health Commission of the People's Republic of China. Notice of the general office of the National Health Commission on the Internet diagnosis and treatment consulting services in epidemic prevention and control. https ://www.nhc.gov.cn/yzygj/s7653p/202002/ec5e345814e744398c2a def17b657fb8.shtml. Accessed 6 Apr 2020

3. Vitale A, Volk ML, Pastorelli D et al (2010) Use of sorafenib in patients with hepatocellular carcinoma before liver transplantation: A cost-benefit analysis while awaiting data on sorafenib safety. Hepatology 51(1):165-173. https://doi.org/10.1002/ hep. 23260

4. Kudo M, Finn RS, Qin S et al (2018) Lenvatinib versus sorafenib in first-line treatment of patients with unresectable hepatocellular carcinoma: a randomised phase 3 non-inferiority trial. Lancet 391(10126):1163-1173. https://doi.org/10.1016/S0140 $-6736(18) 30207-1$

5. Wang Z, Wang J, He J (2020) Active and Effective Measures for the Care of Patients With Cancer During the COVID-19 Spread in China. JAMA Oncol. https://doi.org/10.1001/jamao ncol.2020.1198 (Published online April 01 2020)

Publisher's Note Springer Nature remains neutral with regard to jurisdictional claims in published maps and institutional affiliations. 